\title{
Monochromatic Infrared Photo Energy in Diabetic Peripheral Neuropathy
}

\author{
Tarek A. Ammar \\ Faculty of Physical Therapy, Cairo University, Ahmed Elzaiat St., Ben Elsaryat, Giza 12612, Egypt \\ Correspondence should be addressed to Tarek A. Ammar, tarekphysical@yahoo.com
}

Received 1 May 2012; Accepted 4 June 2012

Academic Editors: B. Dugue and A. Ozcan Edeer

Copyright () 2012 Tarek A. Ammar. This is an open access article distributed under the Creative Commons Attribution License, which permits unrestricted use, distribution, and reproduction in any medium, provided the original work is properly cited.

Diabetes is a very common cause of peripheral neuropathy, and there is no optimal intervention universally accepted by clinicians. Monochromatic infrared photo energy is a relatively new modality used in the United States for reducing pain and increasing circulation. This study investigated the effects of monochromatic infrared photo energy on reducing pain, improving sensation, and increasing balance in patients with diabetic peripheral neuropathy. Thirty-five patients with diabetic peripheral neuropathy completed the program and were randomly assigned into two groups. Group 1 (experimental, $n=18$ ) received monochromatic infrared photo energy, therapeutic exercises, and balance training. Group 2 (control, $n=17$ ) received therapeutic exercises and balance training. Both groups received three treatment sessions per week for 4 weeks. Outcome included pain intensity measured on a visual analogue scale, sensation measured with the Semmes-Weinstein monofilament 5.07, and balance measured with the Berg score, before and after the 12 therapy sessions ( 1 month after the start of the intervention). Analysis of covariance tests revealed statistically significant improvements, specifically, $P=.01, .014$, and .0001 , for pain, sensation, and balance, respectively, in the experimental group. Within the limitations of this study, monochromatic infrared photo energy may play a role in treating diabetic peripheral neuropathy by reducing pain, improving sensation, and increasing balance.

\section{Introduction}

Diabetic peripheral neuropathy (DPN) is a common progressive complication of diabetes mellitus [1], and it may result, in part, from microvascular dysfunction. DPN represents a huge economic burden to the health care system and is prevalent worldwide [2]. Peripheral neuropathy is also linked to substantial reductions in the quality of life, and the condition is associated with diabetes mellitus and other metabolic diseases, chemotherapy, alcohol abuse, infection, environmental toxins or drugs, scar tissue formation following surgery or radiation, as well as idiopathic causes $[3,4]$. Approximately $15 \%$ of the population over 40 years of age experiences peripheral neuropathy, and, in those with diabetes, the prevalence is approximately $29 \%$ [5]. Of these patients, about $50 \%$ are insensitive to the 5.07 SemmesWeinstein monofilament (SWM) at two or more of six measured plantar sites [5]. Despite the prevalence of DPN, many patients are asymptomatic and therefore do not seek care for it [5].

Patients with early DPN usually experience pain that worsens at night: perceived numbness, and a tingling sensation in the feet and hands. Patients may show reduced ability to detect temperature changes and/or pressure in the feet in advanced stages, and these symptoms are associated with postural instability, loss of leg and foot strength, and reduced proprioceptive thresholds in foot inversion, eversion, plantar flexion, and dorsiflexion [6]. Therefore, patients with DPN often develop gait and balance dysfunction that leads to an increased risk of falling, foot ulcers, and amputations. Consequently, patients are often encouraged to use compensatory strategies such as walking aids (cane or walker), and learn about palliative and protective foot care in an effort to identify potential environmental hazards that could lead to pedal cutaneous compromise or injury. 
Although there is no definitive intervention for the treatment of DPN, the mainstay generally hinges on rigorous glycemic control and reduction of pain and paresthesia by either topical or systemic means [6]. Therefore, there is a need for a complementary approach to help improve circulation and reduce pain to be used along with medications.

Various types of electrotherapy, such as transcutaneous electrical nerve stimulation (TENS), pulsed electromagnetic fields (PEMF), static magnetic field therapy, external muscle stimulation, and frequency-modulated electromagnetic neural stimulation, have been reported to decrease pain and increase circulation. However, results of the studies describing the effects of these modalities on peripheral neuropathy remain controversial, and randomized-controlled studies with larger sample sizes and long followup periods are needed in order to better elucidate the efficacy of these modalities. And, although electrotherapy modalities may help decrease pain [7], they do not restore blood flow, which may limit their ability to improve sensation. Therefore, there is a need for a modality that works to restore blood flow.

Monochromatic infrared photo energy (MIPE) represents another approach to the management of DPN. MIPE was cleared by the United States Food and Drug Administration in 1994 for increasing circulation and reducing pain. MIPE at a wavelength of $890 \mathrm{~nm}$ is produced by an array of 60 gallium aluminum arsenide light-emitting diodes located on flexible pads and the near infrared photo energy is delivered in a noninvasive, drug-free manner. The diode array must be placed in direct contact with the target skin, as MIPE energizes cells in the epidermis and the most superficial portion of the dermis, thereby warming the skin. The $890 \mathrm{~nm}$ photo energy penetrates the skin enough to be absorbed by hemoglobin in the rete capillary loops in the papillary dermis, rather than just water in the more superficial layers [8]. At $890 \mathrm{~nm}$, MIPE was shown to increase the concentration of plasma nitric oxide in nondiabetic volunteers and increasing the microcirculation $[9,10]$, and it has, in fact, been shown to reduce the incidence of new foot wounds in elderly patients with diabetes mellitus [11].

A few studies have investigated the efficacy of MIPE at reducing pain, improving foot sensation, and increasing balance in patients with peripheral neuropathy. However, we believe that the results of these previous studies were adversely influenced by methodological limitations that threatened the authors' conclusions, including the lack of a control group [12, 13]. Three retrospective observational studies [14-16] showed MIPE to be effective based on chart review. Interestingly, a randomized controlled trial of photo energy used at home failed to show the modality to be therapeutically effective [17]. In that particular study, moreover, patients were trained to use a MIPE machine at home and were evaluated before and after 90 days of treatment. However, patient activities were not controlled and the treatment was not supervised. Based on understanding of the limitations of the previously published investigations, as well as the purported effects of photo energy on DPN, the author undertook the investigation described in this report in an effort to test the research hypothesis that MIPE could be used to reduce pain, improve sensation, and increase balance in patients with DPN.

\section{Participants and Methods}

2.1. Research Design. A randomized controlled study was undertaken with participants randomly assigned to one of two intervention groups: group 1 received MIPE, therapeutic exercises, and balance training, whereas the participants in group 2 received therapeutic exercises and balance training without MIPE. The duration of intervention was 4 weeks per participant, and each participant was scheduled to undergo 3 therapy sessions per week. Measurements were taken at baseline and after end of treatment ( 4 weeks).

2.2. Participants. Participants were recruited from an outpatient rehabilitation setting and were treated between December 2010 and April 2011. To be included in the study, participants had to have diabetes mellitus, either type 1 or type 2 . The maximum allowable $\mathrm{HbAlc} \mathrm{level} \mathrm{required} \mathrm{for} \mathrm{inclusions}$ was $\leq 7 \%$. Furthermore, to be included, participants' drug regimen, as well as interventions to promote blood flow in the lower extremities, had to remain stable for one month prior to commencement of the investigation, and throughout the course of the investigation once the study commenced. A 1-month washout period was also required for any participant taking any drug aimed at promoting lower extremity arterial perfusion. Still further, to be included, participants had to have DPN as evidenced by insensitivity to the 5.07 SWM on at least 2 of 5 (great toe, fourth toe, and 3 of the 5 metatarsal heads) plantar surfaces of both feet.

The Berg balance score (BBS) [18] was used to measure balance, and patients with scores ranging from 21 to 40 (medium fall risk) were considered eligible to participate in the study. Patients were excluded from the study if they had a history of knee or back surgery, or malignancy. All potential subjects signed a consent form permitting the use of their data for research purposes, and confidentiality was assured by the use of an anonymous coding system. The consent form also included a clear explanation of the benefits and expected possible risks of the study, and the rights of human subjects were protected at all times.

Participants who met the inclusion criteria were randomly assigned to one of the two treatment groups. The randomization process involved blank folders numbered from 1 to 100 and containing hidden codes for group assignment, and a random-number generator had determined the codes. When a participant was eligible and gave consent to participate, the investigator drew the next folder from the file, which determined treatment allocation. Each participant was then tested using a visual analogue pain rating scale, the 5.07 SWM, and the BBS.

An independent investigator, blinded to group allocation, conducted the testing procedures. This investigator assessed participants in both groups at both the initial and final sessions. After initial testing, participants began the intervention on the same day. A licensed physical therapist performed all interventions with the participants from both groups. All participants underwent 3 sessions per week for 4 weeks. 
A coworker helped procure data used in this investigation by taking measurements following the study protocol, although that individual did not devise the study or participate in the analysis or interpretation of the data.

2.3. Intervention. MIPE intervention was administered using the Anodyne Therapy System, model 480 (Anodyne Therapy, LLC, Tampa, FL). The device consisted of a base power unit and 8 therapy pads, each containing 60 gallium aluminum arsenide diodes. The area of light-emitting diodes per therapy pad was $22.5 \mathrm{~cm}^{2}$, yielding a total intervention area of $180 \mathrm{~cm}^{2}$. The diodes delivered MIPE pulsed at $292 \mathrm{~Hz}$ at a wavelength of $890 \mathrm{~nm}$ and provided an energy density of 62.4 Joules $/ \mathrm{cm}^{2}$ [8]. The participants in group 1 received MIPE for 30 to 40 minutes per treatment session, and 4 therapy pads were placed on each lower extremity. The therapist placed one pad at the medial and lateral aspect of each leg immediately above the ankle, and one on the plantar and one on the dorsal surface of each foot. Each subject sat comfortably in a quiet room at $21^{\circ} \mathrm{C}$, and the skin of the intervention area was covered with plastic wrap as a barrier between the skin and the diodes to ensure compliance with infection control procedures. The energy setting on the device was preset at 10 bars for every patient, in accordance with the manufacturer's recommendations. The diodes and plastic wrap were removed at the end of the treatment session. Intervention with MIPE was followed by a physical therapy exercise program that focused on strengthening and balance training (described below).

Participants in group 2 underwent only the physical therapy that was the same regimen undertaken by the participants in group 1. The physical therapy program included static and dynamic balance retraining, as well as active lower extremity strengthening (hip extensors, hip abductors, hip adductors, quadriceps, ankle dorsiflexors) and stretching of the hip, knee, and ankle flexor musculature. Participants in both groups were educated as to the rationale for the therapy, and they received verbal and written instructions related to the proper method of exercise, and they demonstrated to the treating therapist their ability to properly perform the prescribed exercises. All participants were instructed to exercise at home on the days that they did not go to the clinic for supervised intervention, and the home program was monitored by asking the participants to record exercise using weekly self-reported exercise logs.

2.4. Outcome Measurements. The $10 \mathrm{~cm}$ visual analogue pain rating scale was used to measure neuropathic pain because it is reliable and provides a valid assessment of pain intensity [19]. Light touch sensation was assessed with use of the 5.07 SWM [20], which is generally accepted as an effective, inexpensive, portable, painless, easy to administer, and reliable screening method for assessing touch-pressure sensation in a valid fashion. In fact, the SWM was shown to be more sensitive than the vibration perception threshold in measuring peripheral sensation $[21,22]$. The tester asked blindfolded participants to indicate by stating the word-now when and where on their foot they sensed the pressure of the monofilament. Ten specific anatomic sites were tested with the monofilament, including (1) the dorsal midfoot, (2-4) the plantar aspect of the pulp of the first, third, and fifth toes, (5-7) the plantar aspect of the first, third, and fifth metatarsal heads, and (8-10) the medial and lateral aspects of the midfoot (midtarsal joint) and the calcaneus. The tester pressed the monofilament at each site until the filament was grossly observed to bend [20]. The assessor added the number of sites recognized by the participant, with a maximum of 10 and a minimum of zero. Since previous research [23] has shown that monofilaments have variable accuracy and durability with significant reduction of the loading force required to bend the filament after repetitive loading, we replaced the monofilaments after assessing every 10 participants.

Balance was assessed using the BBS, which has been shown to be reliable (intraclass interrater reliability correlation coefficient $=.99$ ) [18]. The BBS tests static and dynamic balance activities and grades 14 items on a scale that ranges from 0 to 4 . A score between 21 and 40 indicates medium balance impairment and fall risk. While a score below 20 indicates high balance impairement and fall risk. A score between 41 and 56 indicates low balance impairement and fall risk. The BBS testing involves the use of chairs with and without arms, a stopwatch, a ruler, and a 6-inch step, and the test must be completed within a 15-minute time limit [18]. It has been suggested that the BBS is the single best predictor of the risk of falling [24]. These outcome measurements were obtained a baseline, prior to intervention, and again at 4 weeks following the intervention.

2.5. Data Management and Analysis. Data analysis was performed using SPSS for Windows, version 18.0. Data were analyzed using the analysis of covariance (ANCOVA), with the pretest (baseline) scores for the outcomes or interest as the covariates. The ANCOVA was used to take into account the baseline measurements for each patient. When baseline information is available, this provides a more precise estimate of the treatment effect than either raw outcomes or change scores [25]. Analyses of covariance were performed to determine whether there is a difference between the two groups on the posttest scores of pain as measured by the visual analogue scale, light touch as measured by the 5.07 SWM, and balance as measured by the BBS. Demographic characteristics of the participants were described in a statistical fashion, and the Bonferroni adjustment and the statistical significance was defined at the $1.6 \%(P \leq .016)$ level.

\section{Results}

A total of 41 patients met the inclusion criteria, including $18(43.9 \%)$ males and $23(56.1 \%)$ females. Of these, 10 (24.39\%) had type 1 diabetes mellitus, and 31 (75.61\%) had type 2 . Random allocation placed $21(51.22 \%)$ into group 1 (to receive MIPE + training) and $20(48.78 \%)$ into group 2 (training only). In group $1(n=21)$, there were $10(47.62 \%)$ males and $11(52.38 \%)$ females, and in group $2(n=20)$, there were $8(40 \%)$ males and $12(60 \%)$ females. Three (14.29\%) participants withdrew from the MIPE group, 1 $(4.76 \%)$ due to the inability to arrange transportation (in 
TABLe 1: Physical characteristics of the participants (number $=35$ participants) $*$.

\begin{tabular}{lcc}
\hline Variable & Group 1-training $+\operatorname{MIPE}^{\wedge}(n=18)$ & Group 2-training only $(n=17) P$ value $^{\dagger}$ \\
\hline Age $($ years $)$ & $62.03 \pm 11.01$ & $59.4 \pm 8.51$ \\
Height $(\mathrm{cm})$ & $161.34 \pm 6.21$ & $158.23 \pm 5.82$ \\
Body weight $(\mathrm{kg})$ & $68.32 \pm 10.19$ & $73.1 \pm 9.61$ \\
Body mass index $\left(\mathrm{kg} / \mathrm{m}^{2}\right)$ & $24.25 \pm 7.21$ & $21.8 \pm 4.14$ \\
\hline
\end{tabular}

${ }^{*}$ Values are mean \pm standard deviation.

${ }^{\wedge}$ MIPE: monochromatic infrared photo energy.

${ }^{\dagger}$ Independent sample $t$-test revealed no statistically significant differences between the treatment groups, as would be expected with random allocation of the intervention.

essence, another scheduling problem), and $1(4.76 \%)$ due to scheduling difficulties, $1(4.76 \%)$ due to the development of congestive heart failure (CHF). In the training only group, $3(15 \%)$ participants withdrew, 2 (10\%) due to scheduling difficulties ( 1 was too busy with work, and another had to take care of a sick relative), and 1 (5\%) for reasons that we could not ascertain.

The loss of participants to follow up was associated with difficulties primarily related to scheduling the intervention sessions in both groups (2 (9.52\%) of 21 in the MIPE group, and $2(10 \%)$ of 20 in the training only group), and for scheduling conflicts in 2 other participants (the $1(4.76 \%)$ who developed CHF in the MIPE group and the $1(5 \%)$ lost for unknown reasons in the training only group). For these reasons, a total of 35 participants, 18 (51.43\%) in the MIPE group and $17(48.57 \%)$ in the training only group, were included in the final analysis.

Baseline demographic characteristics describing the participants who completed the investigation are depicted in Table 1. As expected with random allocation of the intervention, there were no statistically significant differences between the treatment groups in regard to age, height, body weight, and body mass index $(P>.05)$. Data were normally distributed. Mean values and standard deviations of pain intensity, sensation score, and balance score at baseline and at 4 weeks are presented in Table 2. Table 2 depicts the results of dependent samples $t$-tests comparing baseline to 4 week outcomes for pain, monofilament sensation, and Berg balance scores, within the intervention groups. These results showed significant differences in all dependent variables before and after intervention in both groups $(P \leq .05)$. No adverse events were observed or reported by any participant in either intervention group.

Table 3 depicts the results of the ANCOVA, which showed statistically significant differences between the intervention groups relative to pain $\left(F_{1,32}=8.16, P=.01\right)$, sensation $\left(F_{1,32}=4.2, P=.014\right)$, and balance $\left(F_{1,32}=12.06, P=\right.$ $.0001)$. The MIPE group displayed lower mean posttest pain scores, fewer sites of pedal insensitivity as measured with the 5.07 SWM, and higher mean Berg balance scores.

\section{Discussion}

All of the participants in this investigation showed reduction in pain, increased foot sensation, and increased balance scores, in both intervention groups, although the improvements were statistically significantly greater in the group of participants that received MIPE (Tables 2 and 3). In regard to pedal sensation, there was a decrease in number of sites insensitive to the 5.07 SWM in both intervention groups. In the MIPE+ training group, the mean number of insensate sites was $1.4 \pm 2.1$ after 4 weeks, compared to $7.2 \pm 1.8$ at baseline, and this difference was statistically significant $(P=$ .025). In the training only group, the mean number of insensate sites was $7.2 \pm 1.3$ after 4 weeks, compared to $8.3 \pm 0.9$ at baseline, and this difference was not statistically significant $(P=.06)$.

The basic idea of this study was to treat the two groups exactly the same in every detail except one (MIPE). The author examined the two groups to see if the MIPE made a difference between them. The difference between groups was attributed to the MIPE.

Although the exact mechanism by which MIPE improves sensation in the diabetic neuropathic foot is not precisely known, it has been proposed that it leads to increased release of nitric oxide and improved microcirculation for the following reasons

(1) Nobel Laureate Robert Furchgott reported that photo energy modulates circulation, and it has been shown that exposure to $890 \mathrm{~nm}$ near infrared photo energy promotes increased blood flow for several hours in rats by mediating endothelial nitric oxide synthase [26].

(2) Photo energy absorbed by hemoglobin increases the amount of nitric oxide in red blood cells, in the form of nitrosothiols, and therefore MIPE is likely to increase vasodilatation secondary to release of nitric oxide [27].

(3) Diabetic glycosylated hemoglobin binds nitric oxide and inhibits its release from hemoglobin at microcirculatory sites, and MIPE is likely to enable release of nitric oxide from glycosylated hemoglobin [28].

Since patients with DPN often have concomitant decreased capillary blood flow to tissues of the feet and impaired circulation to the peripheral nerves, it is plausible that improved oxygenation and nutrition related to nitric oxide metabolism related to MIPE could promote nerve growth and reestablish nerve membrane potentials that had been reduced by the hypoxic conditions associated with diabetes [29]. Moreover, physical therapy methods and exercise are often used to decrease pain and increase balance in patients with DPN. In a rat model, it was shown that exercise 
TABLE 2: Outcomes at baseline and 4 weeks, by intervention group (number $=35$ participants $)^{*}$.

\begin{tabular}{|c|c|c|c|}
\hline Outcome & & Group 1-training $+\operatorname{MIPE}^{\wedge}(n=18)$ & Group 2-training only $(n=17)$ \\
\hline \multirow{2}{*}{$10-\mathrm{cm}$ analogue pain scale } & Baseline & $6.2 \pm 2.1$ & $7.3 \pm 1.1$ \\
\hline & 4 weeks & $3.9 \pm 1.8$ & $5.1 \pm 2.3$ \\
\hline$P$ value $^{\dagger}$ & & & \\
\hline \multirow{2}{*}{ Monofilament sensation" } & Baseline & $7.2 \pm 1.8$ & $8.3 \pm 0.9$ \\
\hline & 4 weeks & $1.4 \pm 2.1$ & $7.2 \pm 1.3$ \\
\hline$P$ value $^{\dagger}$ & & .025 & .06 \\
\hline \multirow{2}{*}{ Berg balance score ${ }^{\S}$} & Baseline & $31 \pm 9.27$ & $28.58 \pm 10.16$ \\
\hline & 4 weeks & $47.61 \pm 10.16$ & $32.52 \pm 9.54$ \\
\hline$P$ value $^{\dagger}$ & & & \\
\hline
\end{tabular}

* Values are mean \pm standard deviation.

${ }^{\wedge}$ MIPE: monochromatic infrared photo energy.

${ }^{\dagger}$ Dependent sample $t$-test revealed statistically significant differences within the treatment groups except within the second group difference for the sensation measurement.

" Possible score ranging from 0 to 10, indicative of the number of separate pedal anatomic sites where 5.07-monofilament touch-pressure was not appreciated by the participant (lower score indicative of more sensation).

$\S$ A score of 21-40 indicates balance impairment and a heightened medium risk of falling.

TABLE 3: Results of analysis of covariance between the groups at 4 weeks after intervention (number $=35$ patients)* .

\begin{tabular}{|c|c|c|c|c|}
\hline Outcome & Group 1-training $+\operatorname{MIPE}^{\wedge}(n=18)$ & Group 2-training only $(n=17)$ & $F$ statistic $^{\dagger}$ & $P$ value $^{\dagger}$ \\
\hline $10-\mathrm{cm}$ analogue pain scale & $3.9 \pm 1.8$ & $5.1 \pm 2.3$ & 8.16 & .01 \\
\hline Monofilament sensation" & $1.4 \pm 2.1$ & $7.2 \pm 1.3$ & 4.2 & .014 \\
\hline Berg balance score ${ }^{\S}$ & $47.61 \pm 7.39$ & $32.52 \pm 9.54$ & 12.06 & .0001 \\
\hline
\end{tabular}

${ }^{*}$ Values are mean \pm standard deviation.

${ }^{\wedge}$ MIPE: monochromatic infrared photo energy.

${ }^{\dagger}$ Analysis of covariance.

" Possible score ranging from 0 to 10 , indicative of the number of separate pedal anatomic sites where 5.07-monofilament touch-pressure was not appreciated by the participant (lower score indicative of more sensation).

$\S$ A score of 21-40 indicates balance impairment and a medium risk of falling.

could reduce pain by increasing the production of endogenous analgesics [30].

There has been some research into the efficacy of exercise as it pertains to increasing balance in patients with DPN [31]. For example, a meta-analysis concluded that exercise reduced the risk of falling and improved balance in the elderly [32]. However, that particular study did not include those with DPN. In another meta-analysis, investigators found that physical therapy interventions led to minimal improvements in balance or reduction in fall risk in the elderly and those with distal neuropathy, and the authors of that report concluded that patients continued to experience deficits in balance and sensation after the intervention [33].

Moreover, Kruse et al. [34] conducted a 12-month randomized controlled study to investigate the effects of exercise and walking intervention on balance, lower-extremity strength, and fall incidence in 79 patients with DPN. The training included leg strengthening and balance exercises, and the authors of that study did not find statistically significant differences in the incidence of falling between the groups during followup, although they did show a small increase in the amount of time that patients in the intervention group could stand on one leg with their eyes closed at the 1year follow up, which led the investigators to conclude that exercise may increase activity without increasing balance or decreasing the incidence of falling. Interestingly, few authors have found favorable results advocating the use of exercise to increase balance and reduce the risk of falls in people with DPN. Song et al. [35] found that a balance exercise and trunk proprioception program improved balance and trunk proprioception in patients with DPN. They reported statistically significant $(P<.05)$ decreases in postural sway and trunk repositioning errors, and statistically significant increases $(P<.05)$ in dynamic balance using the Berg balance scale, functional reach test, timed up and go test, and $10 \mathrm{~m}$ walking time after balance exercise. Based on the existing literature, we feel that controversy exists in regard to the efficacy of exercise as it relates to improving balance in patients with DPN.

There are a number of published articles [12-17] that focus on the use of MIPE to increase balance, reduce pain, and restore sensation in patients with DPN, although the general conclusion of these reports is that MIPE is a recommended intervention in patients with DPN. Unfortunately, the conclusions of the existing literature are threatened by numerous methodological shortcomings. Leonard et al. [12] investigated the effects of MIPE in regard to sensation, pain, and balance in 18 diabetic patients with DPN, and measured outcomes in terms of the 5.07 monofilament and the modified Michigan neuropathy screening instrument 
obtained before the first and seventh visits, and after the twelfth visit. Although these investigators showed improved outcomes with MIPE, their conclusions were threatened by the lack of a control group. Another investigation [13] considered the effect of MIPE along with other physical therapy interventions in 38 patients with peripheral neuropathy due to diabetes, alcohol abuse, and peripheral vascular disease. $\mathrm{He}$ assessed foot sensation and balance using the 5.07 monofilament and the Tinetti assessment tool, and observed improved sensation, increased balance, and reduced fall risk at the end of 12 sessions and at the 3-month followup. The major limitation of their study was, once again, the lack of a control group, and they did not take into consideration the potential influence that psychoactive drugs may have had on the risk of falling, other medications, or comorbidities (stroke, other neuropathies) which may have had on your participants.

A third report [14] aimed to evaluate the responses of 252 patients with DPN to a health status questionnaire by phone interview following the end of MIPE intervention in patients $>64$ years of age, the participants having been identified from insurance billing records of two providers who used monochromatic energy devices for use at home. After 1 year of followup, they found a reduced incidence of falls (78\%) and fear of falling (79\%) at 1 month, and increased daily living activities (72\%), although the findings of this investigation were limited by recall and ascertainment biases-related patient memory.

A fourth report [15] described decreased pain, improved sensation, and increased balance in 2239 diabetic patients who received MIPE, balance and strengthening exercises in a group of outpatient physical therapy centers, although their findings were limited by the retrospective nature of the investigation, as well as biases related to possible (and likely) insurance coding errors. In a fifth previously published report [16], researchers performed a retrospective study to assess the effect of MIPE and therapeutic exercises in regard to pain reduction, 5.07 monofilament sensation, and Tinetti balance scores in 272 patients (mean age $69 \pm 12.3$ years) with peripheral neuropathy due to a variety of etiologies, treated at 8 different outpatient physical therapy centers. They also reported statistically significant beneficial effects related to the intervention, including a $38 \%$ decrease in pain, $77 \%$ improvement in sensation, and $73 \%$ decrease in balance deficits, although the validity of the results is also threatened by the same limitations that jeopardized the findings of the previously mentioned investigations.

Finally, a sixth published report [17] described a randomized, sham-controlled clinical trial, wherein MIPE was shown to have no effect in reducing pain, improving foot sensation, or increasing quality of life in 60 patients with DPN. In that investigation, the participants received MIPE at home for 40 minutes daily over 90 consecutive days, via 4 pads (dorsal and plantar foot, medial and lateral aspects of the calf). The participants were trained to use the photo energy machine at home, and to log their use, and they were checked after 2 weeks of therapy to verify that the intervention was being used correctly.
Potential limitations of this particular report include the usual problems associated with patients logging their activities, and other biases related to unmeasured confounders. Loss to follow up was mostly due to scheduling difficulties or taking the time to participate in the study in each group. Therefore, it cannot be argued that participants withdrew due to the interventions. There are a number of potential biases that could threaten the validity or conclusions, and for these reasons we realize that future investigation remains necessary in order to better understand the clinical value of MIPE in the management of DPN.

Perhaps the biggest limitation of this study relates to the fact that the author did not employ sham MIPE, and the improvements in the dependent variables could have been due to the placebo effect. Both groups received therapeutic exercises and balance training which may have influenced the improvement. Moreover, the author did not undertake an explanatory analysis, nor did we take into consideration every independent variable that experienced clinicians may think of as important in regard to the treatment of DPN. For instance, the participants were not asked to change any aspect of diet, exercise, drugs, and the author did not analyze the potential influence of psychoactive drugs and other intrinsic risks for falling. Moreover, the neuropathic pain questionnaire could have been used instead of the visual analogue score to measure neuropathic pain [36].

Still further, additional research is needed to more precisely identify the role that nitric oxide plays in these outcomes, and whether or not the improvement in sensation, pain, and balance that were observed in this 1-month follows-up study is sustained longterm. Treatment only lasted for 4 weeks which is too short and it is questionable whether improvement would be lasting. It is also recommend comparing MIPE to other photo energy modalities such as laser to establish its superiority over these modalities. Based on the results of this randomized, controlled clinical trial, MIPE may be effective in decreasing pain, restoring sensation, and increasing balance in patients with DPN.

\section{Conflict of Interests}

The author declares that there is no conflict of interests.

\section{References}

[1] E. B. Cole, "Diabetic peripheral neuropathic pain: recognition and management," Pain Medicine, vol. 8, supplement 2, pp. S27-S32, 2007.

[2] S. H. Ko and B. Y. Cha, "Diabetic peripheral neuropathy in type 2 diabetes mellitus in Korea," Journal of Diabetes \& Metabolism, vol. 36, no. 1, pp. 6-12, 2012.

[3] E. García-Morales, J. L. Lázaro-Martínez, D. MartínezHernández, J. Aragón-Sánchez, J. V. Beneit-Montesinos, and M. A. González-Jurado, "Impact of diabetic foot related complications on the health related quality of Life (HRQol) of patients-a regional study in Spain," International Journal of Lower Extremity Wounds, vol. 10, no. 1, pp. 6-11, 2011.

[4] K. Cheer, C. Shearman, and E. B. Jude, "Managing complications of the diabetic foot," BMJ, vol. 339, no. 7733, pp. 13041307, 2009. 
[5] E. W. Gregg, P. Sorlie, R. Paulose-Ram et al., "Prevalence of lower-extremity disease in the U.S. adult population $\geq 40$ years of age with and without diabetes: 1999-2000 National Health and Nutrition Examination Survey," Diabetes Care, vol. 27, no. 7, pp. 1591-1597, 2004.

[6] S. Tesfaye, "Advances in the management of diabetic peripheral neuropathy," Current Opinion in Supportive and Palliative Care, vol. 3, no. 2, pp. 136-143, 2009.

[7] K. Pieber, M. Herceg, and T. Patemostro-Sluga, "Electrotherapy for the treatment of painful diabetic peripheral neuropathy: a review," Journal of Rehabilitation Medicine, vol. 42, no. 4, pp. 289-295, 2010.

[8] T. Burke, "Questions and answers about the MIRE treatment," Advances in Skin \& Wound Care, vol. 12, no. 1, pp. 369-371, 2003.

[9] L. R. Horwitz, T. J. Burke, and D. Carnegie, "Augmentation of wound healing using monochromatic infrared energy. Exploration of a new technology for wound management," Advances in Wound Care, vol. 12, no. 1, pp. 35-40, 1999.

[10] M. C. Mak and G. L. Cheing, "Immediate effects of monochromatic infrared energy on microcirculation in healthy subjects," Photomedicine and Laser Surgery, vol. 30, no. 4, pp. 193-199, 2012.

[11] M. W. Powell, D. E. Carnegie, and T. J. Burke, "Reversal of diabetic peripheral neuropathy and new wound incidence: the role of MIRE," Advances in skin \& wound care, vol. 17, no. 6, pp. 295-300, 2004.

[12] D. R. Leonard, M. H. Farooqi, and S. Myers, "Restoration of sensation, reduced pain, and improved balance in subjects with diabetic peripheral neuropathy: a double-blind, randomized, placebo-controlled study with monochromatic nearinfrared treatment," Diabetes Care, vol. 27, no. 1, pp. 168-172, 2004.

[13] A. B. Kochman, "Monochromatic infrared photo energy and physical therapy for peripheral neuropathy: influence on sensation, balance, and falls," Journal of Geriatric Physical Therapy, vol. 27, no. 1, pp. 16-19, 2004.

[14] M. W. Powell, D. H. Carnegie, and T. J. Burke, "Reversal of diabetic peripheral neuropathy with phototherapy (MIRE) decreases falls and the fear of falling and improves activities of daily living in seniors," Age and Ageing, vol. 35, no. 1, pp. 11-16, 2006.

[15] L. B. Harkless, S. DeLellis, D. H. Carnegie, and T. J. Burke, "Improved foot sensitivity and pain reduction in patients with peripheral neuropathy after treatment with monochromatic infrared photo energy-MIRE," Journal of Diabetes and its Complications, vol. 20, no. 2, pp. 81-87, 2006.

[16] W. Volkert, A. Hassan, M. A. Hassan et al., "Effectiveness of monochromatic infrared photo energy and physical therapy for peripheral neuropathy: changes in sensation, pain, and balance-a preliminary, multi-center study," Physical and Occupational Therapy in Geriatrics, vol. 24, no. 2, pp. 1-17, 2006.

[17] L. A. Lavery, D. P. Murdoch, J. Williams, and D. C. Lavery, "Does anodyne light therapy improve peripheral neuropathy in diabetes? A double-blind, sham-controlled, randomized trial to evaluate monochromatic infrared photoenergy," Diabetes Care, vol. 31, no. 2, pp. 316-321, 2008.

[18] K. Berg, S. Wood-Dauphinee, J. I. Williams, and D. Gayton, "Measuring balance in the elderly: preliminary development of an instrument," Physiotherapy Canada, vol. 41, no. 6, pp. 304-311, 1989.

[19] S. V. Scrimshaw and C. Maher, "Responsiveness of visual analogue and McGill pain scale measures," Journal of Manipulative and Physiological Therapeutics, vol. 24, no. 8, pp. 501-504, 2001.

[20] M. H. Haloua, I. Sierevelt, and W. J. Theuvenet, "Semmesweinstein monofilaments: influence of temperature, humidity, and age," Journal of Hand Surgery, vol. 36, no. 7, pp. 11911196, 2011.

[21] Y. Feng, F. J. Schlösser, and B. E. Sumpio, "The Semmes Weinstein monofilament examination as a screening tool for diabetic peripheral neuropathy," Journal of Vascular Surgery, vol. 50, no. 3, pp. 675-682, 2009.

[22] A. Mythili, K. D. Kumar, K. A. V. Subrahmanyam, K. Venkateswarlu, and R. G. Butchi, "A comparative study of examination scores and quantitative sensory testing in diagnosis of diabetic polyneuropathy," International Journal of Diabetes in Developing Countries, vol. 30, no. 1, pp. 43-48, 2010.

[23] S. Kumar, D. J. S. Fernando, A. Veves, E. A. Knowles, M. J. Young, and A. J. M. Boulton, "Semmes-Weinstein monofilaments: a simple, effective and inexpensive screening device for identifying diabetic patients at risk of foot ulceration," Diabetes Research and Clinical Practice, vol. 13, no. 1-2, pp. 6368, 1991.

[24] A. Shumway-Cook, M. Baldwin, N. L. Polissar, and W. Gruber, "Predicting the probability for falls in community-dwelling older adults," Physical Therapy, vol. 77, no. 8, pp. 812-819, 1997.

[25] S. Senn, Statistical Issues in Drug Development, John Wiley \& Sons, Chichester, UK, 1997.

[26] Y. Maegawa, T. Itoh, T. Hosokawa, K. Yaegashi, and M. Nishi, "Effects of near-infrared low-level laser irradiation on microcirculation," Lasers in Surgery and Medicine, vol. 27, pp. 427-437, 2000.

[27] M. I. Djibladze, Z. G. Melildshvili, and S. D. Uchaneishvili, "Lasertherapy by noncoherent light field of radiation," Biomedical Sciences Instrumentation, vol. 34, pp. 235-239, 1997.

[28] J. V. Boykin, "The nitric oxide connection: hyperbaric oxygen therapy, becaplermin, and diabetic ulcer management," Advances in Skin \& Wound Care, vol. 13, no. 4, pp. 169-174, 2000.

[29] O. C. Aszmann, K. M. Kress, and A. L. Dellon, "Results of decompression of peripheral nerves in diabetics: a prospective, blinded study," Plastic and Reconstructive Surgery, vol. 106, no. 4, pp. 816-822, 2000.

[30] N. J. Stagg, H. P. Mata, M. M. Ibrahim et al., "Regular exercise reverses sensory hypersensitivity in a rat neuropathic pain model: Role of endogenous opioids," Anesthesiology, vol. 114, no. 4, pp. 940-948, 2011.

[31] K. I. Ites, E. J. Anderson, M. L. Cahill, J. A. Kearney, E. C. Post, and L. S. Gilchrist, "Balance interventions for diabetic peripheral neuropathy: a systematic review," Journal of Geriatric Physical Therapy, vol. 34, no. 3, pp. 109-116, 2011.

[32] C. Sherrington, J. C. Whitney, S. R. Lord, R. D. Herbert, R. G. Cumming, and J. C. T. Close, "Effective exercise for the prevention of falls: a systematic review and meta-analysis," Journal of the American Geriatrics Society, vol. 56, no. 12, pp. 22342243, 2008.

[33] E. E. Hill-Westmoreland, K. Soeken, and A. M. Spellbring, "A meta-analysis of fall prevention programs for the elderly: how effective are they?" Nursing Research, vol. 51, no. 1, pp. 1-8, 2002.

[34] R. L. Kruse, J. W. LeMaster, and R. W. Madsen, "Fall and balance outcomes after an intervention to promote leg strength, balance, and walking in people with diabetic peripheral neuropathy: "feet first" randomized controlled trial," Physical Therapy, vol. 90, no. 11, pp. 1568-1579, 2010. 
[35] C. H. Song, J. S. Petrofsky, S. W. Lee, K. J. Lee, and J. E. Yim, "Effects of an exercise program on balance and trunk proprioception in older adults with diabetic neuropathies," Diabetes

Technology and Therapeutics, vol. 13, no. 8, pp. 803-811, 2011.

[36] S. J. Krause and M. M. Backonja, "Development of a neuropathic pain questionnaire," Clinical Journal of Pain, vol. 19, no. 5, pp. 306-314, 2003. 


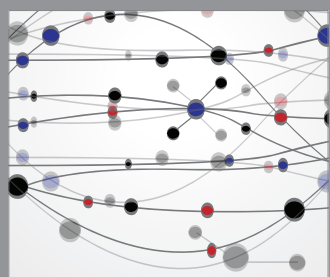

The Scientific World Journal
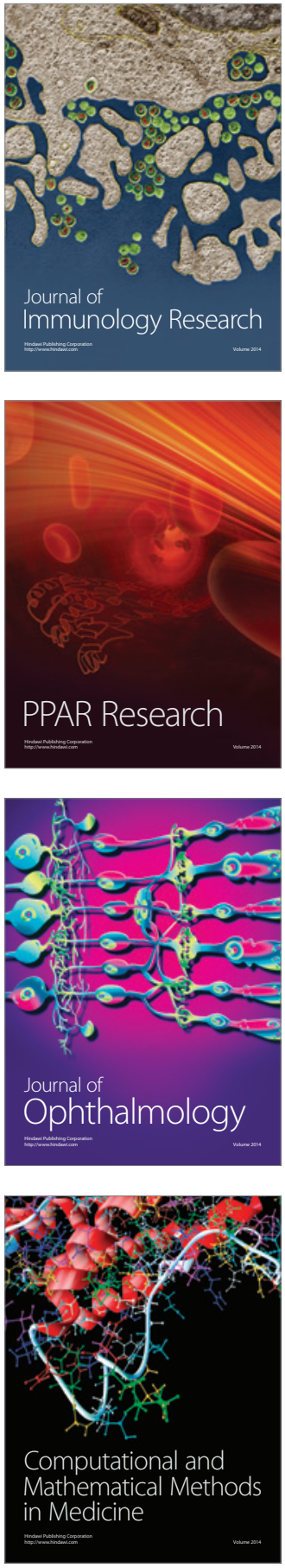

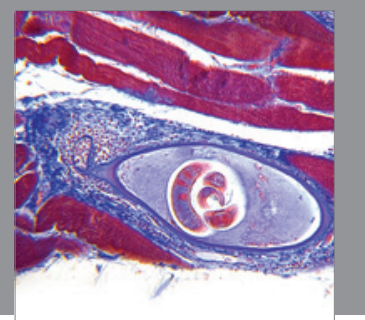

Gastroenterology

Research and Practice
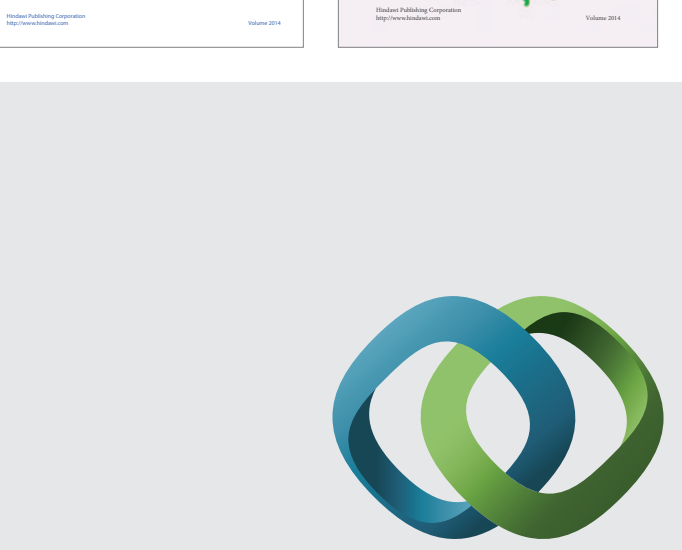

\section{Hindawi}

Submit your manuscripts at

http://www.hindawi.com
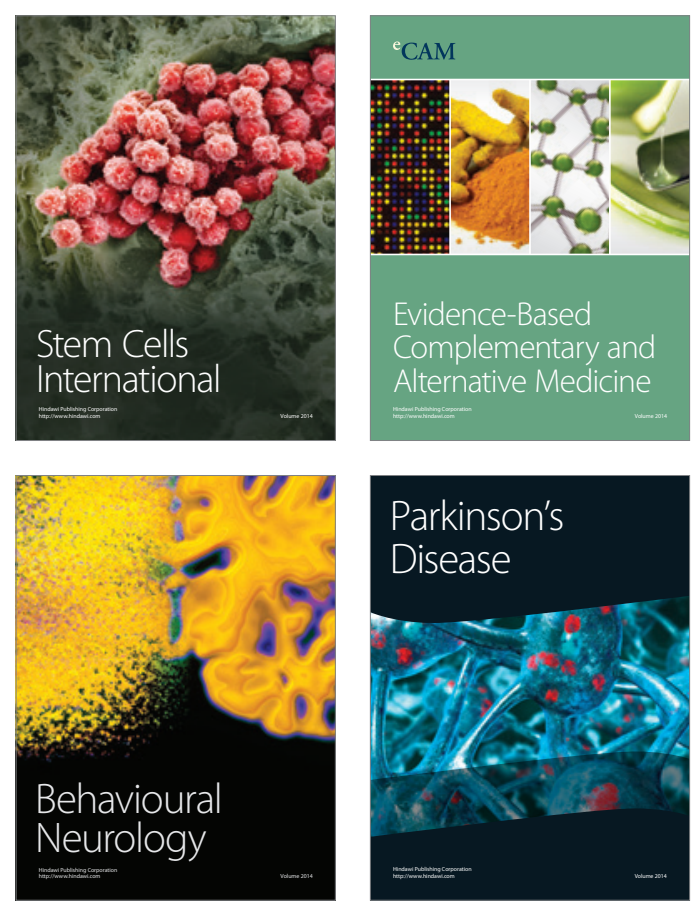

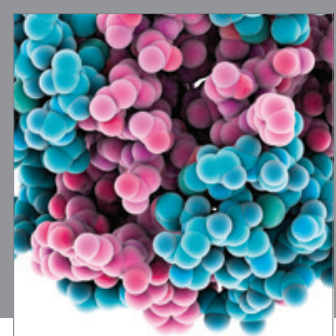

Journal of
Diabetes Research

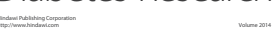

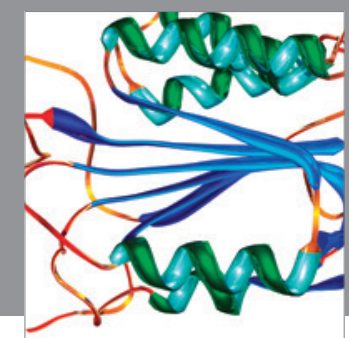

Disease Markers
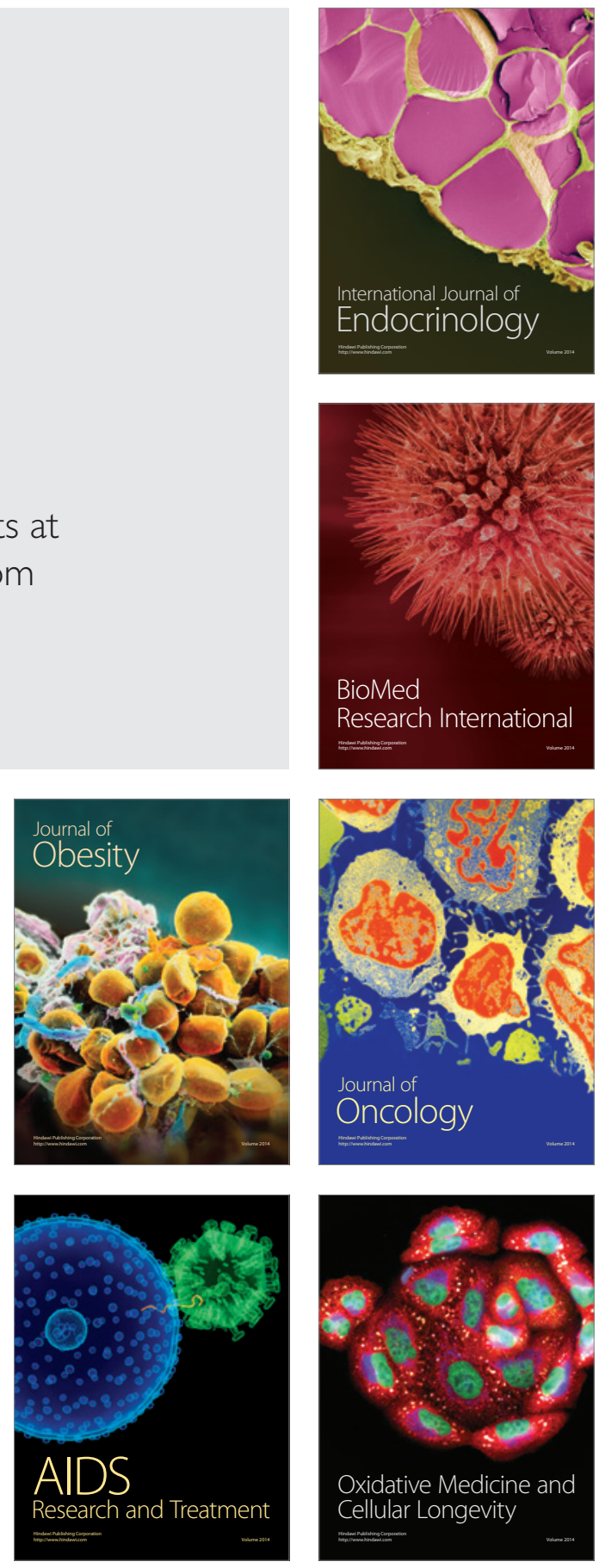